\title{
Fenofibrate-associated changes in renal function and relationship to clinical outcomes among individuals with type 2 diabetes: the Action to Control Cardiovascular Risk in Diabetes (ACCORD) experience
}

\author{
D. E. Bonds • T. E. Craven • J. Buse • J. R. Crouse • \\ R. Cuddihy • M. Elam • H. N. Ginsberg • K. Kirchner • \\ S. Marcovina • J. C. Mychaleckyj • P. J. O'Connor • \\ J.-A. Sperl-Hillen
}

Received: 28 September 2011 / Accepted: 3 February 2012 /Published online: 27 March 2012

(C) Springer-Verlag (outside the USA) 2012

\begin{abstract}
Aims/hypothesis Fenofibrate has been noted to cause an elevation in serum creatinine in some individuals. Participants in the Action to Control Cardiovascular Risk in Diabetes Lipid Study were studied to better characterise who is at risk of an increase in creatinine level and to determine
\end{abstract}

D. E. Bonds $(\bowtie)$

Division of Cardiovascular Sciences, National Heart, Lung and Blood Institute, National Institutes of Health,

Rockledge Center 2, MSC 7936, 6701 Rockledge Drive,

Suite 10018,

Bethesda, MD 20892-7936, USA

e-mail: bondsde@nhlbi.nih.gov

\section{T. E. Craven}

Department of Biostatistical Sciences, Division of Public Health

Sciences, Wake Forest School of Medicine,

Winston-Salem, NC, USA

\section{J. Buse}

Department of Medicine,

University of North Carolina School of Medicine,

Chapel Hill, NC, USA

\section{J. R. Crouse}

Department of Medicine,

Wake Forest School of Medicine,

Winston-Salem, NC, USA

\section{R. Cuddihy}

International Diabetes Center,

Minneapolis, MN, USA

\section{Elam}

Veteran's Affairs Medical Center,

Memphis, TN, USA whether those with creatinine elevation have a differential risk of adverse renal or cardiovascular outcomes.

Methods A fenofibrate-associated creatinine increase (FACI) was defined as an increase in serum creatinine of at least $20 \%$ from baseline to month 4 in participants assigned to fenofibrate. Baseline patient characteristics,

H. N. Ginsberg

Department of Medicine,

Columbia University College of Physicians and Surgeons,

New York, NY, USA

K. Kirchner

Montgomery Veterans Administration Medical Center,

Jackson, MS, USA

S. Marcovina

Northwest Lipid Metabolism and Diabetes Research Laboratory,

University of Washington,

Seattle, WA, USA

J. C. Mychaleckyj

Department of Public Health Sciences, University of Virginia,

Charlottesville, VA, USA

P. J. O'Connor · J.-A. Sperl-Hillen

HealthPartners Research Foundation,

Minneapolis, MN, USA

Present Address:

R. Cuddihy

US Medical Affairs of sanofi-aventis,

Bridegwater, NJ, USA 
and baseline and 4-month drug, clinical, laboratory characteristics and study outcomes were examined by FACI status. Results Of the sample, $48 \%$ of those randomised to receive fenofibrate had at least a $20 \%$ increase in serum creatinine within 4 months. In multivariable analysis, participants who were older, male, used an ACE inhibitor at baseline, used a thiazolidinedione (TZD) at 4 months post-randomisation, had baseline CVD, and had lower baseline serum creatinine and LDL-cholesterol levels were all more likely to meet the criteria for FACI. Participants in the FACI group were also more likely to have a decrease in their serum triacylglycerol level from baseline to 4 months. No differences in study outcomes were seen by FACI criteria.

Conclusions/interpretation Several characteristics predict a rapid rise in serum creatinine upon starting fenofibrate. Participants who met the criteria for FACI also had a greater change in triacylglycerol levels. In the setting of careful renal function surveillance and reduction of fenofibrate dose as indicated, no increase in renal disease or cardiovascular outcome was seen in those individuals demonstrating FACI.

\section{Trial registration: ClincalTrials.gov: NCT00000620}

Funding: The ACCORD Trial was supported by grants (N01HC-95178, N01-HC-95179, N01-HC-95180, N01-HC-95181, N01-HC-95182, N01-HC-95183, N01-HC-95184, IAA-Y1HC-9035 and IAA-Y1-HC-1010) from the National Heart, Lung, and Blood Institute; by the National Institute of Diabetes and Digestive and Kidney Diseases, the National Institute on Aging, and the National Eye Institute; by the Centers for Disease Control and Prevention; by General Clinical Research Centers and by the Clinical and Translational Science Awards. Abbott Laboratories, Amylin Pharmaceutical, AstraZeneca Pharmaceuticals LP, Bayer HealthCare LLC, Closer Healthcare, GlaxoSmithKline Pharmaceuticals, King Pharmaceuticals, Merck, Novartis Pharmaceuticals, Novo Nordisk, Omron Healthcare, sanofi-aventis US and Takeda Pharmaceuticals provided study medications, equipment or supplies.

Keywords Cardiovascular - Creatinine - Fenofibrate . Outcomes $\cdot$ Renal

\begin{tabular}{|c|c|}
\hline \multicolumn{2}{|c|}{ Abbreviations } \\
\hline ACCORD & $\begin{array}{l}\text { Action to Control Cardiovascular Risk in } \\
\text { Diabetes }\end{array}$ \\
\hline CKD-EPI & Chronic kidney disease-epidemiology \\
\hline CVD & Cardiovascular disease \\
\hline DAIS & Diabetes Atherosclerosis Intervention Study \\
\hline eGFR & Estimated GFR \\
\hline FACI & Fenofibrate-associated creatinine increase \\
\hline FIELD & $\begin{array}{l}\text { Fenofibrate Intervention and Event Lowering } \\
\text { in Diabetes }\end{array}$ \\
\hline PPAR & Peroxisome proliferator-activated receptors \\
\hline ZD & Thiazolidinedione \\
\hline
\end{tabular}

Abbreviations

ACCORD Action to Control Cardiovascular Risk in Diabetes

CKD-EPI Chronic kidney disease-epidemiology

Cardiovascular disease

DAIS Diabetes Atherosclerosis Intervention Study

Estimated GFR

FACI Fenofibrate-associated creatinine increase

Fenofibrate Intervention and Event Lowering

PPAR Peroxisome proliferator-activated receptors

\section{Introduction}

Fibrates, including fenofibrate, have been used as lipidaltering medications for more than four decades. Although the US Food and Drug Administration has recently issued a communication regarding the lack of incremental benefit of fenofibrate over statin monotherapy with regard to the prevention of CVD [1], it remains an important drug for use in reducing triacylglycerol levels and is the most commonly prescribed fibrate in both the USA and Canada [2].

As early as 1999, fenofibrate was noted to cause an elevation in serum creatinine in some individuals shortly after starting the drug [3]. Broeders et al [4] reviewed the data from seven smaller studies on fenofibrate (144 patients, $200 \mathrm{mg}$ daily dose) and found that serum creatinine increased from 8 to $18 \%$ from baseline upon starting fenofibrate. The Diabetes Atherosclerosis Intervention Study (DAIS), a clinical trial of the effect of fenofibrate on heart disease in patients with diabetes, reported similar findings [5]. In 2005, the Fenofibrate Intervention and Event Lowering in Diabetes (FIELD) study noted an increase in plasma creatinine of $10-12 \mu \mathrm{mol} / \mathrm{l}$ in the fenofibrate group compared with participants randomised to placebo [6]. Similar results have been found with other drugs in this class, with the possible exception of gemfibrozil [7, 8].

Although the increase in serum creatinine level is welldocumented, patient characteristics and possible drug interactions that may precipitate the increase have not been well characterised. Although initial case reports of increased creatinine with fenofibrate treatment included patients with impaired renal function, renal transplant and proteinuria, previous studies have not determined whether impaired renal function is a predictor of creatinine elevation following fenofibrate therapy $[4,9,10]$. Increased creatinine level has also been observed in those with normal renal function [11]. Ellen et al examined the co-administration of simvastatin and fenofibrate as a predictor of the increase in creatinine and found no additive effect of the two medications [12]. No large study has systematically examined the predictors of the creatinine increase. Whereas the DAIS and FIELD studies did report fewer participants in the fenofibrate arm progressing to microalbuminuria, this finding has not been examined in other clinical trials [5, 6]. The Action to Control Cardiovascular Risk in Diabetes (ACCORD) Lipid Trial offers an opportunity to better understand the aetiology and consequences of the creatinine increase observed in some patients receiving fenofibrate.

This paper has three aims: first, to determine the characteristics of patients experiencing a rise in creatinine shortly after starting fenofibrate; second, to determine the longitudinal pattern of creatinine elevation in these participants; and third, to determine whether the creatinine elevation predicts a change in renal function, as measured by the albumin/creatinine ratio, or an increased risk of a cardiovascular outcome. 


\section{Methods}

Study design The ACCORD Lipid Study was a $2 \times 2$ factorial design trial conducted with patients with type 2 diabetes. The objective was to determine whether a treatment strategy designed to raise HDL-cholesterol levels and lower triacylglycerol by the addition of fenofibrate to statin therapy would have cardiovascular benefits over a strategy targeting primarily LDL-cholesterol reduction with statin therapy alone. The full details of the study design have been published elsewhere [13].

ACCORD enrolment began January 2001 and was completed in October 2005. The study was sponsored by the National Heart Lung and Blood Institute, with 77 clinical sites in the USA and Canada participating. The protocol was reviewed and approved by the institutional review board or ethics committee of each participating clinical centre, and all participants provided informed consent. All 10,251 ACCORD participants were randomised to either intensive glucose control $\left(\mathrm{HbA}_{1 \mathrm{c}}<6.0 \%\right.$ [42 mmol/mol]) or standard glucose control $\left(\mathrm{HbA}_{1 \mathrm{c}}\right.$ between $7.0 \%$ [53 mmol/mol] and $7.9 \%$ [63 mmol/ mol]). In addition, 5,518 participants were assigned to the Lipid Study and randomised to receive a blinded lipid drug that was either fenofibrate $160 \mathrm{mg}$ or placebo, in addition to open-label simvastatin therapy. Statins were titrated to achieve LDLcholesterol targets for primary and secondary cardiovascular protection, with a maximum dose of $40 \mathrm{mg}$ simvastatin (or equivalent). Other concomitant prescription lipid therapies were not permitted, as per the protocol. The study concluded in June 2009, and resulted in no statistical difference in the primary outcome, a composite of non-fatal myocardial infarction, non-fatal stroke and cardiovascular death. The results of the Lipid Study have previously been reported [14].

Study participants Participants were eligible to enrol in the ACCORD study if they had type 2 diabetes, possessed a $\mathrm{HbA}_{1 \mathrm{c}}$ between $7.5 \%$ [59 mmol/mol] and $11 \%$ [96.72 mmol/ $\mathrm{mol}]$ and were either (a) between the ages of 40 and 79 years with CVD, or (b) between the ages of 55 and 79 with either evidence of subclinical CVD or the presence of two or more additional risk factors for CVD. In addition, to be eligible for the Lipid Study, participants had to meet the following criteria: an estimated off-statin LDL-cholesterol level of 1.55$4.65 \mathrm{mmol} / \mathrm{l}$; an HDL-cholesterol level below $1.42 \mathrm{mmol} /$ 1 for women and black participants, or below $4.5 \mathrm{mmol} / \mathrm{l}$ for all other groups; and a triacylglycerol level below $8.5 \mathrm{mmol} / \mathrm{l}$ if untreated or below $4.52 \mathrm{mmol} / \mathrm{l}$ if the participants were currently taking lipid-lowering medication. Potential participants were excluded from the ACCORD study if their serum creatinine was greater than $132.6 \mu \mathrm{mol} / \mathrm{l}$ owing to the anticipated use of metformin in the trial.

Lipid study procedures Participants were randomised to either statin therapy alone or statin plus fenofibrate therapy using a centralised randomisation procedure and permuted blocks. During the main ACCORD Lipid Trial, all Lipid Study participants were started on open-label simvastatin (during the Vanguard phase, participants were started on statin therapy only if their LDL-cholesterol level was greater than $3.00 \mathrm{mmol} / \mathrm{l}$ ); all Vanguard participants were placed on statin when the main trial period began. One month after randomisation, participants were started on either fenofibrate or a matching placebo tablet. The simvastatin dose was consistent with national guidelines and was modified as the study progressed in response to changing recommendations [15]. During the main study, all Lipid Study participants were provided with either 20 or $40 \mathrm{mg} /$ day of simvastatin based on risk profile and LDL-cholesterol level.

The starting dose of fenofibrate was $160 \mathrm{mg} /$ day. In 2004, procedures were changed to adjust the ongoing dosing of fenofibrate to the estimated GFR (eGFR) using the Modification of Diet in Renal Disease equation [16]. Participants with an $\mathrm{eGFR} \geq 50 \mathrm{ml} \mathrm{min}{ }^{-1} 1.73 \mathrm{~m}^{-2}$ received $160 \mathrm{mg} /$ day of either fenofibrate or matching placebo, while those with an eGFR between 30 and $50 \mathrm{ml} \mathrm{min}{ }^{-1} 1.73 \mathrm{~m}^{2}$ received $54 \mathrm{mg} /$ day of fenofibrate or placebo. If an eGFR fell below $30 \mathrm{ml} \mathrm{min}{ }^{-1} 1.73 \mathrm{~m}^{2}$ on two consecutive occasions, the fenofibrate (but not the statin) was permanently discontinued.

In addition to lipid medications, glucose-lowering medications were also supplied to all Lipid Study participants. A medication inventory of concomitant classes of medication was obtained from participants at baseline and annually.

Designation as showing FACI Lipid Study participants initiating full-dose fenofibrate at least 30 days prior to their 4month serum creatinine assessment were eligible for the study cohort examined in this report. Those participants who also experienced at least a $20 \%$ rise in creatinine above their baseline creatinine value at the first on-medication creatinine measurement (month 4 of the ACCORD study) were classified as having a fenofibrate-associated creatinine increase (FACI).

Laboratory measurements Serum creatinine was measured at baseline and every 4 months throughout the study. If an eGFR fell below $30 \mathrm{ml} \mathrm{min}^{-1} 1.73 \mathrm{~m}^{2}$, participants were asked to undergo a repeat serum creatinine measurement within 2 weeks to confirm the value. Analysis of serum creatinine was performed using the Roche Creatinine Plus enzymatic method (Roche Diagnostics, Basel, Switzerland) on a Modular P analyser. The method is based on conversion of the creatinine present in a sample to sarcosine using creatininase and creatinase enzymes. The results obtained by this procedure are traceable to the isotope dilution mass spectrometry reference method. The sensitivity of the Creatinine Plus assay is $2.29 \mu \mathrm{mol} / \mathrm{l}$. The intra-assay $\mathrm{CVs}$ based on an analysis of low- and high-quality control samples were 0.8 and $0.7 \%$, respectively, whereas the interassay CVs were 1.6 and $2.5 \%$, respectively. 
Urine was collected at baseline, at 2 years and 4 years, and at the end of the study from all participants. Serum and urine creatinine were determined enzymatically on a Roche Double Modular P Analytics automated analyser. Interassay precision is consistently $<1.4 \%$ for the high-quality and $<2.2 \%$ for the low-quality control samples. Urine albumin was determined by immunonephelometry on a Siemens BN II nephelometer (Siemens Healthcare Diagnostics, Tarrytown, NY, USA). The sensitivity of the assay is $1.6 \mathrm{~g} / 1$ with interassay CVs of 3.0, 2.6 and $4.9 \%$ for control levels of $8.9 \mathrm{~g} / 1,66 \mathrm{~g} / 1$, and $161 \mathrm{~g} / \mathrm{l}$, respectively. All serum and urine samples were analysed on the day of sample receipt. Microalbuminuria was defined as an albumin/creatinine ratio of $\geq 3.5 \mathrm{mg} / \mathrm{mmol}$ but $<35 \mathrm{mg} / \mathrm{mmol}$ in a random urine sample, and macroalbuminuria was defined as an albumin/creatinine ratio $\geq 35 \mathrm{mg} / \mathrm{mmol}$.

Statistical analysis Descriptive statistics (means and SDs of continuous factors, and frequencies and percentages of categorical factors) were computed by FACI status, and groups were compared using univariable logistic regression models. Analyses of factors related to FACI were limited to participants assigned to fenofibrate treatment. Relationships were examined between FACI and (1) baseline demographic and medical characteristics; (2) baseline and early (4-month) ontreatment medication use; and (3) early changes in clinical measures.

Factors with a demonstrated or postulated relationship to renal function on fenofibrate were considered. A 'best' multivariable logistic regression model to predict FACI status was constructed using a best subsets variable selection procedure based on the score statistic from fitted models. All variables presented in Tables 1,2 and 3 below were considered as candidates. The top five models of each size up to 20 covariates were considered. The model with the smallest Akaike Information Criterion and all candidate variables significant at the 0.05 alpha-level were chosen. The relationship between FACI status and baseline to end-of-study change in urinary albumin/creatinine ratio was assessed by analysis of covariance of the within-subject differences in the $\log _{10}$ transformation of the ratios. Relationships between FACI status and time to follow-up renal outcome (incident micro- and macroalbuminuria) and to fatal/non-fatal CVD event were assessed using proportional hazards regression models. Each model examined included factors selected in the final logistic regression model for FACI to control for potential confounders. Distributions of the temporal change in renal function were examined using box-and-whisker plots of the follow-up minus baseline difference in serum creatinine and eGFR [17]. All analyses were performed using SAS version 9.2 software (SAS Institute Inc., Cary, NC, USA).

\section{Results}

A total of 5,518 participants were enrolled in the ACCORD Lipid Study, with 2,765 randomised to receive fenofibrate plus statin and 2,753 to receive placebo plus statin. The baseline creatinine (mean \pm SD) was $70.92 \pm 17.54 \mu \mathrm{mol} / 1$ in the fenofibrate arm and $70.92 \pm 17.54 \mu \mathrm{mol} / 1$ in the placebo arm. At the 4-month visit, the serum creatinine had increased to $83.12 \pm 23.64 \mu \mathrm{mol} / 1$ in the fenofibrate arm and was unchanged $(70.16 \pm 17.54 \mu \mathrm{mol} / \mathrm{l})$ in the placebo arm. This difference was statistically significant $(p<0.0001)$. Among participants assigned to fenofibrate treatment, 2,523 (91\%) were placed on full-dose medication at least 30 days prior to the 4-month serum creatinine assessment. Of those, 1,212 participants $(48 \%)$ met the criteria for FACI $(>20 \%$ increase in serum creatinine at the 4-month follow-up visit) compared with $223(9 \%)$ of placebo patients. Subsequent to the 4-month creatinine measurement, 2,462 (98\%) remained active in the
Table 1 Association of baseline demographic and medical characteristics with FACI $>20 \%$ at month 4 of the study

Values are $\%(n)$ or mean (SD)

INT, intensive arm of glycaemia study

${ }^{\mathrm{a}} \mathrm{OR}$ for $1 \mathrm{SD}$ increase for continuous factors

${ }^{\mathrm{b}}{ }_{n=1,205}$ for FACI and

1,304 for non-FACI

${ }^{\mathrm{c}} n=1,202$ for FACI and 1,298 for non-FACI

\begin{tabular}{lllll}
\hline Characteristic & $\begin{array}{l}\text { FACI } \\
(n=1,212)\end{array}$ & $\begin{array}{l}\text { No FACI } \\
(n=1,311)\end{array}$ & $\begin{array}{l}\text { Unadjusted OR } \\
(95 \% \text { CI })^{\mathrm{a}}\end{array}$ & $p$ value \\
\hline Randomised to INT (\%) & $53(638)$ & $45(593)$ & $1.35(1.15,1.57)$ & 0.0002 \\
Age (years) & $62(7)$ & $62(7)$ & $1.05(0.97,1.13)$ & 0.3 \\
Female (\%) & $28(1,212)$ & $32(422)$ & $0.80(0.68,0.95)$ & 0.01 \\
Race/ethnicity (\%) & $69(841)$ & $64(843)$ & & 0.08 \\
Non-Hispanic white & $13(152)$ & $14(186)$ & $0.82(0.65,1.04)$ & \\
African-American & $7(80)$ & $9(111)$ & $0.72(0.53,0.98)$ & \\
Hispanic & $6(75)$ & $7(85)$ & $0.88(0.64,1.22)$ & \\
Asian & $5(64)$ & $7(86)$ & $0.75(0.53,1.05)$ & \\
Other & $33(393)$ & $28(367)$ & $1.24(1.04,1.47)$ & 0.01 \\
Microalbuminuria ${ }^{\mathrm{b}}(\%)$ & $8(99)$ & $6(75)$ & $1.47(1.07,2.00)$ & 0.02 \\
Macroalbuminuria ${ }^{\mathrm{b}}(\%)$ & $11(8)$ & $10(7)$ & $1.12(1.03,1.22)$ & 0.006 \\
Duration of diabetes (years) $^{\mathrm{c}}$ & $38(464)$ & $34(444)$ & $1.21(1.03,1.43)$ & 0.02 \\
History of clinical CVD (\%) & & & & \\
\hline
\end{tabular}


Table 2 Association of medication use at baseline and month 4 of the study, and the change in use from baseline to month 4 of the study with FACI $>20 \%$ at month 4 of the study
Values are $\%(n)$

${ }^{\mathrm{a}} n=1,154$ for FACI and 1,199 for no-FACI

${ }^{\mathrm{b}} n=1,192$ for FACI and 1,290 for no-FACI

\begin{tabular}{lllll}
\hline Characteristic & $\begin{array}{l}\text { FACI } \\
(n=1,212)\end{array}$ & $\begin{array}{l}\text { No FACI } \\
(n=1,311)\end{array}$ & $\begin{array}{l}\text { Unadjusted } \\
\text { OR }(95 \% \text { CI })\end{array}$ & $p$ value \\
\hline Baseline medications (\%) & & & & \\
$\quad$ Insulin & $33(402)$ & $34(441)$ & $0.98(0.83,1.16)$ & 0.8 \\
ACE inhibitor & $57(693)$ & $50(656)$ & $1.33(1.14,1.56)$ & 0.0003 \\
Angiotensin-receptor blocker & $13(163)$ & $15(202)$ & $0.85(0.68,1.07)$ & 0.2 \\
TZD & $18(223)$ & $17(228)$ & $1.07(0.87,1.31)$ & 0.5 \\
Aspirin & $59(712)$ & $57(748)$ & $1.07(0.91,1.26)$ & 0.4 \\
Loop diuretic & $11(127)$ & $8(100)$ & $1.42(1.08,1.87)$ & 0.01 \\
Thiazide diuretic & $29(354)$ & $25(325)$ & $1.25(1.05,1.49)$ & 0.01 \\
Potassium-sparing diuretic & $2(29)$ & $3(36)$ & $0.87(0.53,1.43)$ & 0.6 \\
Statin & $63(765)$ & $56(736)$ & $1.34(1.14,1.57)$ & 0.0004 \\
Non-steroidal anti-inflammatory drug & $3(30)$ & $2(32)$ & $1.01(0.61,1.68)$ & 0.9 \\
Medications at month 4 of study $(\%)$ & & & & \\
$>20$ mg simvastatin $^{\mathrm{a}}$ & $5(55)$ & $4(43)$ & $1.35(0.90,2.02)$ & 0.2 \\
Insulin $^{\mathrm{b}}$ & $45(533)$ & $42(545)$ & $1.11(0.94,1.30)$ & 0.2 \\
TZD & $59(707)$ & $44(570)$ & $1.84(1.57,2.16)$ & $<0.0001$ \\
\hline
\end{tabular}

Lipid Trial. Of the 1,191 active participants in the FACI group, $316(26 \%)$ were placed on a reduced dose of fenofibrate and
419 (35\%) stopped active medication at some point after the 4-month visit. Among 1,271 active participants in the group

Table 3 Association of clinical measures at baseline and the change from baseline to month 4 of the study, with FACI $>20 \%$

\begin{tabular}{|c|c|c|c|c|c|c|}
\hline \multirow[t]{2}{*}{ Characteristic } & \multicolumn{2}{|c|}{ FACI $(n=1,212)$} & \multicolumn{2}{|c|}{ No FACI $(n=1,311)$} & \multirow{2}{*}{$\begin{array}{l}\text { Unadjusted OR } \\
(95 \% \mathrm{CI})\end{array}$} & \multirow[t]{2}{*}{$p$ value } \\
\hline & $n$ & $\begin{array}{l}\text { Mean (SD) } \\
\text { or \% (num) }\end{array}$ & $n$ & $\begin{array}{l}\text { Mean (SD) } \\
\text { or num (\%) }\end{array}$ & & \\
\hline \multicolumn{7}{|l|}{ At enrollment into study: } \\
\hline $\mathrm{HbA}_{1 \mathrm{c}}(\%)$ & 1,211 & $8.3(1.0)$ & 1,307 & $8.3(1.0)$ & $1.00(0.92,1.08)$ & 0.9 \\
\hline $\mathrm{HbA}_{1 \mathrm{c}}(\mathrm{mmol} / \mathrm{mol})$ & 1,211 & $67(13)$ & 1,307 & $67(13)$ & $1.00(0.92,1.08)$ & 0.9 \\
\hline Serum creatinine $(\mu \mathrm{mol} / \mathrm{l})$ & 1,212 & $78.68(19.45)$ & 1,299 & $83.98(20.33)$ & $0.78(0.71,0.84)$ & $<0.0001$ \\
\hline Serum creatinine $>76 \mu \mathrm{mol} / 1$ at baseline $(\%)$ & 1,212 & $20(240)$ & 1,299 & $30(386)$ & $0.58(0.49,0.70)$ & $<0.0001$ \\
\hline CKD-EPI eGFR $\left(\mathrm{ml} \mathrm{min}{ }^{-1} 1.73 \mathrm{~m}^{2}\right)$ & 1,212 & $86.4(16.3)$ & 1,299 & $82.0(17.1)$ & $1.31(1.21,1.42)$ & $<0.0001$ \\
\hline $\log _{10}[$ urinary albumin/creatinine ratio $(\mathrm{mg} / \mathrm{mmol})]$ & 1,205 & $0.39(0.67)$ & 1,304 & $0.32(0.62)$ & $1.08(1.02,1.13)$ & 0.004 \\
\hline LDL-cholesterol (mmol/l) & 1,212 & $2.51(0.78)$ & 1,299 & $2.64(0.80)$ & $0.84(0.77,0.91)$ & $<0.0001$ \\
\hline HDL-cholesterol (mmol/1) & 1,212 & $0.98(0.21)$ & 1,299 & $0.98(0.21)$ & $0.95(0.88,1.03)$ & 0.2 \\
\hline Triacylglycerol (mmol/l) & 1,212 & $2.21(1.37)$ & 1,299 & $2.09(1.15)$ & $1.10(1.02,1.19)$ & 0.01 \\
\hline Triacylglycerol $>2.30 \mathrm{mmol} / 1$ and $\mathrm{HDL} \leq 0.88 \mathrm{mmol} / 1(\%)$ & 1,212 & $18(219)$ & 1,299 & $17(219)$ & $1.09(0.89,1.34)$ & 0.4 \\
\hline Systolic BP (mmHg) & 1,204 & $134(18)$ & 1,300 & $133(18)$ & $1.05(0.97,1.13)$ & 0.2 \\
\hline Diastolic BP (mmHg) & 1,204 & $74(11)$ & 1,300 & $74(10)$ & $1.00(0.92,1.08)$ & 0.9 \\
\hline \multicolumn{7}{|l|}{ Change: month 4 minus baseline } \\
\hline $\mathrm{HbA}_{1 \mathrm{c}}(\%)$ & 1,162 & $-1.09(1.2)$ & 1,250 & $-1.01(1.2)$ & $0.94(0.86,1.01)$ & 0.1 \\
\hline $\mathrm{HbA}_{1 \mathrm{c}}(\mathrm{mmol} / \mathrm{mol})$ & 1,162 & $-1.09(1.2)$ & 1,250 & $12(10)$ & $0.94(0.86,1.01)$ & 0.1 \\
\hline LDL-cholesterol (mmol/l) & 1,210 & $-0.23(0.88)$ & 1,296 & $-0.26(0.85)$ & $1.03(0.95,1.11)$ & 0.5 \\
\hline HDL-cholesterol (mmol/1) & 1,210 & $0.09(0.20)$ & 1,296 & $0.07(0.18)$ & $1.10(1.02,1.19)$ & 0.02 \\
\hline Triacylglycerols (mmol/l) & 1,210 & $-0.66(1.16)$ & 1,296 & $-0.43(1.21)$ & $0.81(0.74,0.89)$ & $<0.0001$ \\
\hline Systolic BP (mmHg) & 1,095 & $-8(17)$ & 1,027 & $-2(16)$ & $0.67(0.61,0.74)$ & $<0.0001$ \\
\hline Diastolic BP (mmHg) & 1,095 & $-4(10)$ & 1,027 & $-2(9)$ & $0.74(0.67,0.81)$ & $<0.0001$ \\
\hline
\end{tabular}

Values are $\%(n)$ or mean (SD)

${ }^{\mathrm{a}} \mathrm{OR}$ for $1 \mathrm{SD}$ increase for continuous factors 
with $<20 \%$ increase in serum creatinine (no FACI), 235 (18\%) were placed on a reduced dose of fenofibrate, and $327(26 \%)$ stopped active medication at some point after the 4-month visit.

Characteristics predicting creatinine elevation In univariate analysis, several baseline demographic and medical characteristics were associated with meeting the criteria for entry into the FACI group. These are described in Table 1. Briefly, participants were more likely to experience FACI if they were male and non-Hispanic white, had a longer duration of diabetes, had pre-existing kidney disease as defined by the presence of micro- or macroalbuminuria at baseline, were randomised to the intensive glycaemia group, or had a history of clinical CVD at baseline.

Univariable relationships between selected medication use at study entry and at month 4 and FACI at month 4 are described in Table 2. A larger number of participants in the FACI group were treated at baseline with an ACE inhibitor, a loop diuretic, a thiazide diuretic or a statin. We also found that the treatment with a thiazolidinedione (TZD) medication for management of diabetes in the first 4 months of the trial was associated with FACI classification by the 4-month visit.

Univariable relationships between baseline and 4-month clinical characteristics and FACI status at 4 months are shown in Table 3. Participants in the FACI group had a lower average creatinine (higher eGFR) at baseline and a higher $\log _{10}$ urine albumin/creatinine ratio. LDL-cholesterol level was lower in the participants with FACI compared with those without, and triacylglycerol level was higher. No differences were seen in baseline HDL-cholesterol or baseline BP values. Examination of the change in clinical characteristics from baseline to month 4 showed that participants with FACI had a greater mean increase in HDLcholesterol and greater reductions in triacylglycerol levels. Participants in the FACI group also showed greater reductions in systolic and diastolic BP.

In multivariable logistic regression analysis adjusted for factors selected via forward variable selection to predict FACI group status (Table 4), participants with FACI were more likely to be older and male, and have a longer duration of diabetes, a baseline history of CVD, higher triacylglycerol levels, higher systolic BP, and lower baseline levels of serum creatinine and LDL-cholesterol. They were also more likely to experience a greater reduction in triacylglycerol levels and systolic BP during the first 4 months of treatment. On-study use of TZD by month 4 of the ACCORD study also predicted allocation to FACI status.zxz

Long-term renal and cardiovascular outcomes The fenofibrateassociated increase in creatinine and decrease in GFR as estimated by the chronic kidney disease-epidemiology (CKD-EPI) equation [17] was stable over time, as indicated by the box-andwhisker plot of change in Fig. 1. An examination of FACI status vs long-term renal and CVD events, and change in urinary albumin/creatinine ratio is presented in Table 5 . We found no significant differences in relationships between FACI status and incident microalbuminuria, macroalbuminuria or end-stage renal disease, mean change in urine albumin/creatinine ratio, or occurrence of fatal or non-fatal CVD events (the primary outcome of the ACCORD study).
Table 4 Multivariable logistic regression model to predict FACI $>20 \%$ at month 4 of the study

Results of best subsets variable selection procedure using score statistics $(n=2,054 ; 984$ FACI; 1,070 no FACI; max-rescaled $R^{2}=14.9 \%$ [28])

${ }^{\mathrm{a}} \mathrm{OR}$ for $1 \mathrm{SD}$ increase for continuous factors

\begin{tabular}{|c|c|c|}
\hline Variable & $\mathrm{OR}^{\mathrm{a}}(95 \% \mathrm{CI})$ & $p$ value \\
\hline Age (SD change 7 years) & $1.12(1.02,1.24)$ & 0.02 \\
\hline Female & $0.59(0.46,0.74)$ & $<0.0001$ \\
\hline Duration of diabetes (SD change 7.5 years) & $1.11(1.01,1.22)$ & 0.03 \\
\hline Baseline history of CVD & $1.30(1.06,1.59)$ & 0.01 \\
\hline Baseline use of ACE inhibitor & $1.33(1.10,1.60)$ & 0.003 \\
\hline Baseline use of loop diuretic & $1.42(1.01,2.00)$ & 0.04 \\
\hline Baseline use of thiazide diuretic & $1.38(1.11,1.72)$ & 0.003 \\
\hline Baseline serum creatinine (SD change $15.25 \mu \mathrm{mol} / \mathrm{l}$ ) & $0.71(0.64,0.79)$ & $<0.0001$ \\
\hline Baseline LDL-cholesterol (SD change $0.78 \mathrm{mmol} / \mathrm{l}$ ) & $0.86(0.78,0.95)$ & 0.002 \\
\hline Baseline triacylglycerol level (SD change $1.14 \mathrm{mmol} / \mathrm{l}$ ) & $1.39(1.23,1.58)$ & $<0.0001$ \\
\hline Month 4 minus baseline triacylglycerol (SD change $1.27 \mathrm{mmol} / \mathrm{l}$ ) & $0.71(0.62,0.81)$ & $<0.0001$ \\
\hline Baseline systolic BP (SD change $18 \mathrm{mmHg}$ ) & $1.51(1.34,1.70)$ & $<0.0001$ \\
\hline Month 4 minus baseline systolic BP (SD change 17 mmHg) & $0.67(0.60,0.75)$ & $<0.0001$ \\
\hline Change in use of TZD from baseline to month 4 of the study & & $<0.0001$ \\
\hline No baseline TZD, no month 4 TZD & Reference & \\
\hline No baseline TZD, on TZD month 4 & $2.19(1.78,2.69)$ & \\
\hline On baseline TZD, no month 4 TZD & $1.18(0.46,3.03)$ & \\
\hline On baseline TZD, on TZD month 4 & $1.56(1.20,2.03)$ & \\
\hline
\end{tabular}


Fig. 1 Box-and-whisker plots of the distribution of follow-up minus baseline difference in serum creatinine (a) and eGFR (b) for participants without FACI (empty boxes on left at each time point) and with FACI (grey-filled boxes on right at each time point). Rectangles depict the lower quartile (bottom), median (solid line inside box) and upper quartile (top) of the difference. The black dot inside the rectangle shows the mean. Whiskers extend to 1.5 times the interquartile range above and below the rectangles, with grey dots showing individual values beyond 1.5 interquartile ranges below the 25 th or above the 75 th percentiles a
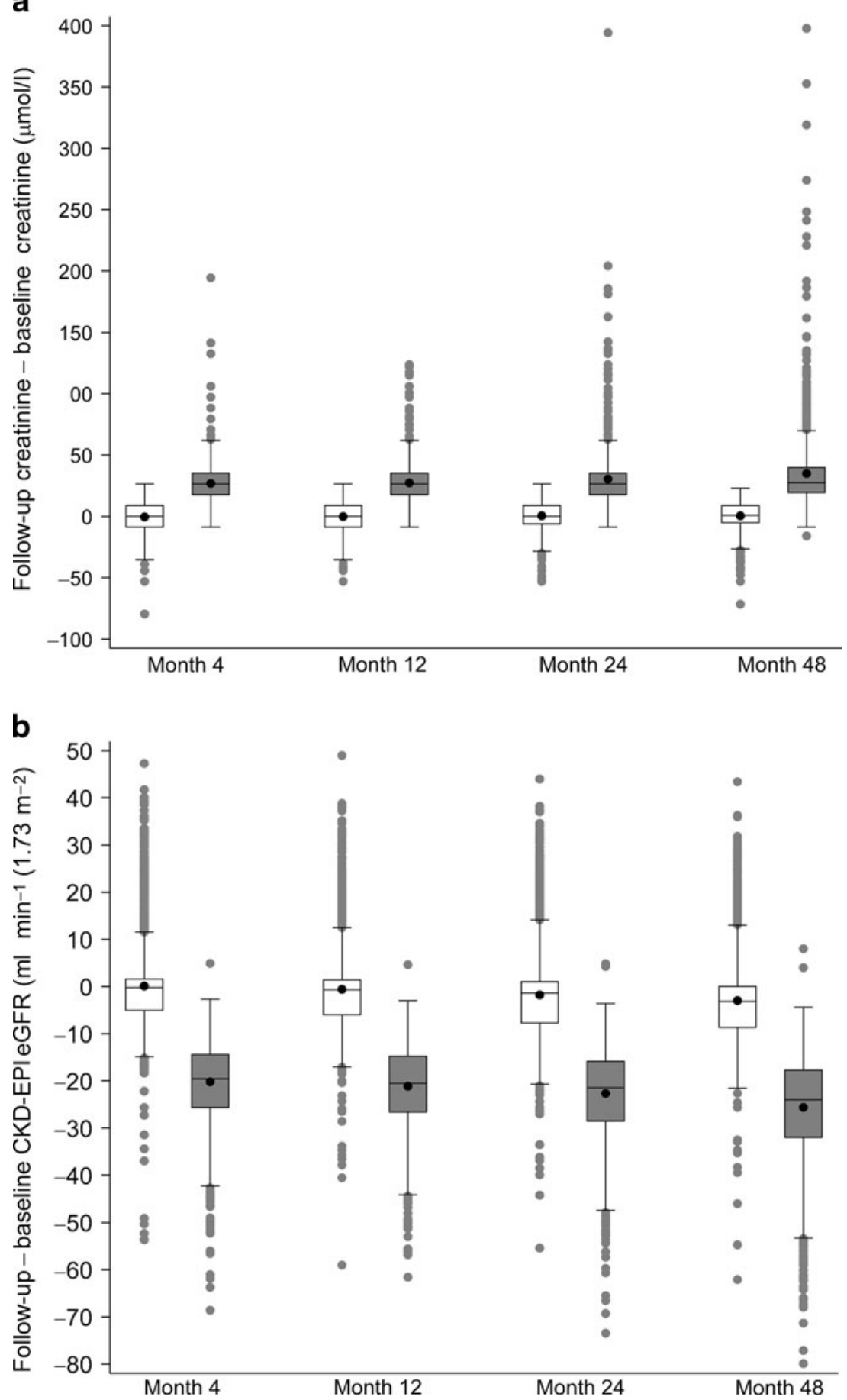

\section{Discussion}

In this epidemiological analysis of the ACCORD Lipid Study participants, we found that $48 \%$ of the participants randomised to receive fenofibrate and $9 \%$ of those randomised to receive placebo had at least a $20 \%$ increase in serum creatinine shortly after starting on blinded study medication. Analysis of the participants in the fenofibrate arm who met this criterion were more likely to be older and male, to have existing CVD, to be taking an ACE inhibitor or a diuretic at the time of starting fenofibrate, to possess lower serum creatinine and LDLcholesterol levels at the time of randomisation, and to be randomised to intensive glycaemic control compared with the participants in the fenofibrate arm who did not experience a $20 \%$ increase in creatinine. Concurrent use of a TZD for blood glucose control was also strongly associated with experiencing an increase in creatinine level. Participants were regularly tested for renal function, and reductions in medications were 
Table 5 Relationships between FACI $>20 \%$ at month 4 of the study, incident renal disease and primary study outcome

\begin{tabular}{|c|c|c|c|c|c|c|}
\hline \multirow[t]{2}{*}{ Outcome } & \multicolumn{2}{|l|}{ FACI } & \multicolumn{2}{|c|}{ No FACI } & \multirow[t]{2}{*}{$\mathrm{HR}^{\mathrm{a}}(95 \% \mathrm{CI})$} & \multirow[t]{2}{*}{$p$ value } \\
\hline & $n$ & Value & $n$ & Value & & \\
\hline Microalbuminuria (\%) & 776 & $21(163)$ & 890 & $24(208)$ & $0.89(0.69,1.13)$ & 0.3 \\
\hline Macroalbuminuria (\%) & 1,051 & $5(55)$ & 1,170 & $6(69)$ & $0.88(0.58,1.34)$ & 0.5 \\
\hline End-stage renal disease $(\%)$ & 1,212 & $3.2(39)$ & 1,311 & $2.7(35)$ & $1.39(0.81,2.40)$ & 0.2 \\
\hline Change in $\log _{10}$ transform of urine albumin to creatinine ratio & 1,027 & $0.004(0.547)$ & 1,049 & $0.079(0.516)$ & - & 0.09 \\
\hline Primary study outcome (follow-up fatal/nonfatal CVD events) (\%) & 1,212 & $10(117)$ & 1,311 & $11(143)$ & $0.84(0.63,1.15)$ & 0.3 \\
\hline
\end{tabular}

Values are \% $(n)$ or mean $(\mathrm{SD})$

${ }^{\mathrm{a}} \mathrm{HR}$ from proportion hazards regression model of time to event. Hazard ratios and $p$ values from model controlling for factors included in Table 4

implemented as indicated. In this context, no significant adverse effects on other measures of renal function were seen during the trial among those who met the FACI criteria. In addition, participants meeting the FACI criteria had the same rate for the ACCORD primary cardiovascular outcome as did those who did not.

Those participants treated with fenofibrate who experienced an increase in creatinine level also showed a greater decrease in serum triacylglycerol level, a greater increase in HDLcholesterol, and greater reductions in both systolic and diastolic BP at month 4 of the study. These greater responses in terms of triacylglycerols, HDL-cholesterol and BP are consistent with the hypothesis that participants in the FACI group were more sensitive to fenofibrate treatment. Additional studies will be necessary to determine whether these individuals can be identified through genetic analyses, and pharmacogenomic studies may be warranted. In this regard, it is interesting to note that the frequency of the peroxisome proliferator-activated receptor (PPAR) alpha $\mathrm{G} / \mathrm{C}$ intron 7 gene polymorphism has been associated with a greater triacylglycerol-lowering response to fenofibrate in patients with type 2 diabetes mellitus [18].

Many of the characteristics that we found to be associated with FACI were not surprising. Medications with a known association with a change renal function were predictive, as were characteristics associated with advanced disease, such as duration of diabetes. Interestingly, we found a strong association of an increase in creatinine level among participants also treated with a TZD. The basis for this finding is unclear. ATZD was used by $74 \%$ of participants in the ACCORD study. Fenofibrate is a PPAR-alpha agonist, and rosiglitazone, the TZD used most often in ACCORD, is a PPAR-gamma agonist. TZDs have been noted to increase creatinine in some patients with diabetes [19], and the combination of a TZD and fenofibrate may have had an additive effect. There does not appear to be any long-term adverse effect on renal function from the combination, a finding that confirms a recent study in diabetesinduced experimental nephropathy [20]. In univariable analysis, individuals randomised to intensive glycaemia treatment were more likely to experience FACI; however, intensive glycaemic treatment was not selected in the final multivariable logistic regression model. Given the association of TZD with FACI, this association is likely to reflect the increased use of TZD in this group [21].

Our results confirm that fenofibrate use increases serum creatinine level surprisingly often. This finding is consistent with those of previous studies $[4,11,22,23]$. The underlying mechanism of the increase is still, however, unclear. Changes in GFR, creatinine excretion within the kidney tubules, altered renal haemodynamics and changes in production of creatinine by skeletal muscle have all been hypothesised [9, 11, 24]. In a crossover study of fenofibrate and placebo, Ansquer et al did not find a significant change in GFR as measured by inulin clearance, tubular creatinine secretion as measured by urinary/plasma creatinine to urinary/plasma inulin ratio, or total creatinine excretion as measured by $24 \mathrm{~h}$ urinary creatinine collection [11]. Other investigations have not documented any changes in renal blood flow as measured by para-aminohippurate [24]. Another possible mechanism is increased production of creatinine from muscle. Hottelart et al [24] did note an increase in the products of muscle breakdown in some patients taking fenofibrate, but this was not correlated with an increase in serum creatinine. Thus, this potential mechanism does not appear to account for the increase seen.

The mechanism(s) underlying increased plasma creatinine with fenofibrate and other PPAR-alpha activators is likely to be complex given the wide tissue distribution and broad range of genes regulated by this transcriptional activator, which may include genes related to creatine synthesis, metabolism and transport [25, 26]. For example, it has been noted that fibrates alter the production of renal eicosanoids, which may affect renal blood flow [4, 27]. It is interesting to note that the concomitant administration of ACE inhibitors and diuretics, agents that are associated with azotaemia due to altered renal blood flow, was increased among patients experiencing FACI while taking fenofibrate. This suggests a common mechanism(s). More work is needed to better understand the mechanisms that underlie this change. 
Preservation of long-term renal function among those randomised to fenofibrate has been previously reported by the FIELD investigators [23]. Davis et al, in a study of renal function among FIELD participants participating in a washout study, found that those participants randomised to fenofibrate had an eGFR closer to baseline values than did participants randomised to placebo [23]. We were able to confirm that, among individuals who showed an increase in creatinine level immediately upon starting fenofibrate, no long-term adverse renal effects or any increased risk of the main study outcome, CVD, was seen. The overall trend was towards a slight protective effect with a smaller change in urinary albu$\mathrm{min} /$ creatinine ratio and fewer participants developing microalbuminuria, although neither of these findings was statistically significant. This relationship may have been somewhat attenuated by the greater number of participants meeting FACI status who had a protocol-mandated reduction in fenofibrate dose or cessation of fenofibrate therapy compared with those who did not meet FACI status. The occurrence of micro- and macroalbuminuria was significantly reduced by fenofibrate treatment in the full ACCORD Lipid Study cohort [14]. These findings suggest that fenofibrate might act via pathways similar to ACE inhibitors, which raise creatinine while being 'reno-protective'. In this regard, it is of interest that treatment with ACE inhibitors at baseline predicted increases in creatinine levels during fenofibrate therapy.

A number of factors limit the interpretation of these data. First, the generalisability of results to individuals without diabetes or others not similar to the ACCORD participants is limited. Second, the defined cut-point of a $20 \%$ rise in serum creatinine is based on clinical considerations and is arbitrary; a different cut-point may have altered the results. Third, we do not have data on other independent markers of renal function such as cystatin for the ACCORD participants and were unable to conduct more rigorous and direct measurements of renal function (e.g. inulin clearance) that could have provided insights into the pathways involved in our findings. Additionally, the risk model's $R^{2}$ value of $14.9 \%$ indicates that there are other determinants governing the development of FACI that are still unknown. Finally, it is possible that, in routine clinical practice, outcomes could be worse if renal surveillance and timely adjustments in fenofibrate doses were carried out less consistently than in the ACCORD research protocol.

These limitations must be balanced against the strengths of the study. This is one of largest clinical trials of fenofibrate, and participants were followed for an extended period of time. The size and duration provide for stable estimates of the creatinine increase. We were able to examine the effects of fenofibrate among those participants who experienced the increase, and found no adverse effect on end-stage renal disease or albuminuria. Finally, we identified factors such as older age, male sex and existing CVD as risk factors for increased serum creatinine level, and found that the risk of creatinine elevation was higher in those treated with TZDs and certain classes of antihypertensive medication.

In conclusion, we have identified clinical characteristics that predict which patients with diabetes treated with fenofibrate will develop an increase in creatinine level. We have also confirmed that no long-term adverse effects are seen on renal function, and that those individuals who experience the increase in creatinine may be more sensitive to the proposed beneficial effects of fenofibrate; they may, for example, show greater clinical benefit as indicated by reductions in serum triacylglycerols and increases in HDL-cholesterol. Participants meeting the FACI criteria who had appropriate adjustments to their medication did not have a greater incidence of renal or cardiovascular endpoints.

Funding The ACCORD Trial was supported by grants (N01-HC95178, N01-HC-95179, N01-HC-95180, N01-HC-95181, N01-HC95182, N01-HC-95183, N01-HC-95184, IAA-Y1-HC-9035, and IAAY1-HC-1010) from the National Heart, Lung, and Blood Institute; by the National Institute of Diabetes and Digestive and Kidney Diseases, the National Institute on Aging and the National Eye Institute; by the Centers for Disease Control and Prevention; by the General Clinical Research Centers; and by the Clinical and Translational Science Awards. Abbott Laboratories, Amylin Pharmaceutical, AstraZeneca Pharmaceuticals LP, Bayer HealthCare LLC, Closer Healthcare, GlaxoSmithKline Pharmaceuticals, King Pharmaceuticals, Merck, Novartis Pharmaceuticals, Novo Nordisk, Omron Healthcare, sanofi-aventis US and Takeda Pharmaceuticals provided study medications, equipment or supplies.

Duality of interest D. E. Bonds, T. E. Craven, J. R. Crouse, K. Kirchner, S. Marcovina, P. J. O'Connor and J.-A. Sperl-Hillen declare that they have no duality of interest associated with this manuscript.

$\mathrm{J}$. Buse is a consultant or investigator under contract with the University of North Carolina with multiple companies; these provide no direct financial benefit to him. They include the following: Amylin Pharmaceutical, Inc.; Andromeda; Bayhill Therapeutics; Biodel; Boehringer Ingelheim; Bristol-Myers Squibb; Catabasis; Diartis; Elcelyx; Eli Lilly and Co.; Exsulin; GI Dynamics; Halozyme, Inc.; HoffmanLaRoche Inc.; Johnson \& Johnson; Lexicon; LipoScience; Medtronic MiniMed, Inc.; Merck; Metabolon; Novan; Novo Nordisk Pharmaceuticals, Inc.; Osiris Therapeutics, Inc.; Orexigen; Pfizer, Inc.; sanofiaventis; Tolerex; Transition Therapeutics; and TransPharma. R. Cuddihy has served as PI or Co-investigator for sponsored clinical trials research at several companies while at the International Diabetes Center: Amylin, Abbott, Bayer, Calibra Medical, Daiichi-Sankyo, Dexcom, Edwards Lifesciences, Eli Lilly, Hygeia, Intarcia, Johnson and Johnson/Lifescan, Mannkind, Medtronic, Merck, Novo Nordisk, Quotient Diagnostics, ResMed, Roche, sanofi-aventis, Schering-Plough, Takeda, Valeritas; he is also Advisory Board Member for Abbott, Bayer, CeQur, Eli Lilly, Novo Nordisk, Roche; and receives support for educational activities from Lifescan, Eli Lilly, Merck, Novartis, sanofi-aventis and ScheringPlough; his spouse is a physician and medical director with Optum Health, a subdivision of United Health Group. All honoraria, speaking fees, consulting fees and research and educational support monies were paid directly to the non-profit International Diabetes Center of which R. Cuddihy was a salaried employee; in 2011 he became an employee of sanofi-aventis, US. M. Elam is a speaker/consultant for Abbott/Merck Schering Plough/Pfizer. H. N. Ginsberg consults with, speaks for and receives a research grant from Merck; he also consults for Abbott. J. C. Mychaleckyj receives a research grant from Abbott. 
Contribution statement DEB was involved in the conception and design of the study, interpretation of the data, drafting of the article and final approval of the version to be published. TEC participated in analysis and interpretation of data, drafting sections of the paper and final approval of the version to be published. JB, JRC, RC, ME, KK, SM, JCM, PJO'C and JAS-H contributed to conception and design of the study, interpretation of the data, revising it critically for important intellectual content and final approval of the version to be published. HNG was involved in conception and design, interpretation of the data, drafting sections of the paper, revising other sections critically for important intellectual content and final approval of the version to be published.

\section{References}

1. Food and Drug Administration (2011) Prescibing information Trilipix. Available from www.accessdata.fda.gov/scripts/cder/drugsatfda/ index.cfm?fuseaction=Search.DrugDetails, accessed 13 December 2011

2. Jackevicius CA, Tu JV, Ross JS, Ko DT, Carreon D, Krumholz HM (2011) Use of fibrates in the United States and Canada. JAMA 305:1217-1224

3. Hottelart C, el Esper N, Achard JM, Pruna A, Fournier A (1999) Fenofibrate increases blood creatinine, but does not change the glomerular filtration rate in patients with mild renal insufficiency. Nephrologie 20:41-44

4. Broeders N, Knoop C, Antoine M, Tielemans C, Abramowicz D (2000) Fibrate-induced increase in blood urea and creatinine: is gemfibrozil the only innocuous agent? Nephrol Dial Transplant 15:1993-1999

5. Ansquer JC, Foucher C, Rattier S, Taskinen MR, Steiner G (2005) Fenofibrate reduces progression to microalbuminuria over 3 years in a placebo-controlled study in type 2 diabetes: results from the Diabetes Atherosclerosis Intervention Study (DAIS). Am J Kidney Dis 45:485-493

6. Keech A, Simes RJ, Barter P et al (2005) Effects of long-term fenofibrate therapy on cardiovascular events in 9795 people with type 2 diabetes mellitus (the FIELD study): randomised controlled trial. Lancet 366:1849-1861

7. Meade TW (2001) Design and intermediate results of the Lower Extremity Arterial Disease Event Reduction (LEADER)* trial of bezafibrate in men with lower extremity arterial disease [ISRCTN4119421]. Curr Contr Trials Cardiovasc Med 2:195-204

8. Rizos E, Bairaktari E, Ganotakis E, Tsimihodimos V, Mikhailidis DP, Elisaf M (2002) Effect of ciprofibrate on lipoproteins, fibrinogen, renal function, and hepatic enzymes. J Cardiovasc Pharmacol Therapeut 7:219-226

9. Lipscombe J, Bargman JM (2001) Fibrate-induced increase in blood urea and creatinine. Nephrol Dial Transplant 16:1515

10. Deighan CJ, Caslake MJ, McConnell M, Boulton-Jones JM, Packard CJ (2001) Comparative effects of cerivastatin and fenofibrate on the atherogenic lipoprotein phenotype in proteinuric renal disease. J Am Soc Nephrol 12:341-348

11. Ansquer JC, Dalton RN, Causse E, Crimet D, Le Malicot K, Foucher C (2008) Effect of fenofibrate on kidney function: a 6-week randomized crossover trial in healthy people. Am J Kidney Dis 51:904-913
12. Ellen RL, McPherson R (1998) Long-term efficacy and safety of fenofibrate and a statin in the treatment of combined hyperlipidemia. Am J Cardiol 81:60B-65B

13. Buse JB, Bigger JT, Byington RP et al (2007) Action to control cardiovascular risk in diabetes (ACCORD) trial: design and methods. Am J Cardiol 99:21i-33i

14. Ginsberg HN, Elam MB, Lovato LC et al (2010) Effects of combination lipid therapy in type 2 diabetes mellitus. N Engl J Med 362:1563-1574

15. Ginsberg HN, Bonds DE, Lovato LC et al (2007) Evolution of the lipid trial protocol of the Action to Control Cardiovascular Risk in Diabetes (ACCORD) trial. Am J Cardiol 99:56i-67i

16. Levey AS, Coresh J, Greene T et al (2006) Using standardized serum creatinine values in the modification of diet in renal disease study equation for estimating glomerular filtration rate. Ann Intern Med 145:247-254

17. Levey AS, Stevens LA, Schmid CH et al (2009) A new equation to estimate glomerular filtration rate. Ann Intern Med 150:604-612

18. Foucher C, Rattier S, Flavell DM et al (2004) Response to micronized fenofibrate treatment is associated with the peroxisomeproliferator-activated receptors alpha $\mathrm{G} / \mathrm{C}$ intron7 polymorphism in subjects with type 2 diabetes. Pharmacogenetics 14: 823-829

19. Stulc T, Kasalova Z, Krejci H, Dolezalova R, Ceska R (2005) Effect of rosiglitazone on homocysteine and creatinine levels in patients with type 2 diabetes. Atherosclerosis 183:367-368

20. Arora MK, Reddy K, Balakumar P (2010) The low dose combination of fenofibrate and rosiglitazone halts the progression of diabetes-induced experimental nephropathy. Eur J Pharmacol 636:137-144

21. Gerstein HC, Miller ME, Byington RP et al (2008) Effects of intensive glucose lowering in type 2 diabetes. N Engl J Med 358:2545-2559

22. Forsblom C, Hiukka A, Leinonen ES, Sundvall J, Groop PH, Taskinen MR (2010) Effects of long-term fenofibrate treatment on markers of renal function in type 2 diabetes: the FIELD Helsinki substudy. Diabetes Care 33:215-220

23. Davis TM, Ting R, Best JD et al (2011) Effects of fenofibrate on renal function in patients with type 2 diabetes mellitus: the Fenofibrate Intervention and Event Lowering in Diabetes (FIELD) Study. Diabetologia 54:280-290

24. Hottelart C, El Esper N, Rose F, Achard JM, Fournier A (2002) Fenofibrate increases creatininemia by increasing metabolic production of creatinine. Nephron 92:536-541

25. Mandard S, Muller M, Kersten S (2004) Peroxisome proliferatoractivated receptor alpha target genes. Cell Mol Life Sci 61:393416

26. Schiffrin EL (2005) Peroxisome proliferator-activated receptors and cardiovascular remodeling. Am J Physiol Heart Circ Physiol 288:H1037-H1043

27. Huang H, Morisseau C, Wang J et al (2007) Increasing or stabilizing renal epoxyeicosatrienoic acid production attenuates abnormal renal function and hypertension in obese rats. Am J Physiol Renal Physiol 293:F342-F349

28. Nagelkerke NJD (1991) A note on a general definition of the coefficient of determination. Biometrika 78:691-692 